\title{
Applications of Supercritical Fluid in Alloplastic Bone Graft: A Novel Method and in Vitro Tests
}

\author{
Ching-Feng Chen, ${ }^{*}$, Che-Shoa Chang, ${ }^{\ddagger}$ Yan-Ping Chen, ${ }^{\S}$ Tien-Sheng Lin," Cheng-Yao Su, ${ }^{\dagger}$ and \\ Sheng-Yang Lee Le $^{\perp}$ \\ Institute of Clinical Dentistry, School of Dentistry, National Yang-Ming University, 155, Li-Nong Street, \\ Section 2, Peitou, Taipei, Taiwan, Republic of China, Department of Dentistry \& Oral-Maxillo-facial Surgery, \\ Taipei Veterans General Hospital, Department of Chemical Engineering, National Taiwan University, Fine \\ Metals Laboratory, Industrial Technology Research Institute, and School of Dentistry, College of Oral \\ Medicine, Taipei Medical University
}

\begin{abstract}
In this study, supercritical fluid (SCF) technology is discussed in relation to biomaterial processing, especially the fabrication and processing of alloplastic bone graft. SCF offers excellent extraction properties for some compounds because of its favorable diffusivity, viscosity, zero surface tension, liquidlike density and solvating power, and other physical properties. The most desirable SCF solvent for extraction is supercritical carbon dioxide $\left(\mathrm{SCCO}_{2}\right)$, which is nontoxic, nonflammable, inexpensive, friendly to mankind, and environmentally benign and has mild supercritical conditions $\left(T_{\mathrm{c}}=31.2^{\circ} \mathrm{C}, P_{\mathrm{c}}=7.386 \mathrm{MPa}\right)$. For the porous structure of porcine vertebra with its content of lipids, proteins, and inorganic substances in the cells and intercellular matrix, $\mathrm{SCCO}_{2}$ combined with ethanol or glutaraldehyde (GA) is used to prepare novel porcine-derived bone grafts in three supercritical settings and one subcritical condition for control comparison. The biocompatibility of these novel bone grafts was tested with human MG63 osteoblast-like cells and mouse fibroblast 3T3 cells by MTT assay. The results revealed that materials processed by $\mathrm{SCCO}_{2}$ combined with both ethanol and GA were not cytotoxic and allowed the differentiation and proliferation of test cells. The good performances of these novel bone grafts can be explained by the characteristics of SCF. Applications of SCF in biomaterials are very promising for bone regeneration and tissue engineering. Additional advanced in vitro and in vivo studies must be performed in the future for optimization.
\end{abstract}

\section{Introduction}

Much research has been performed on bone regeneration. The most traditional way to repair bone damage is by using autografts, because this simultaneously represents the properties of osteogenesis, osteoinduction, and osteoconduction and thus is considered as the most ideal bone graft. However, this approach was demonstrated to carry a $17.9 \%$ complication rate, and its use is restricted by limited source and quantity. ${ }^{1}$ Therefore, the use of allografts and alloplasts has arisen. Hydroxyapatite $(\mathrm{HA})^{2-7}$ and demineralized freeze-dried bone allograft (DFDBA $)^{8-12}$ are the most widely used and studied materials, although they demonstrate the properties of only osteoconduction and osteoinduction, respectively. HA has a high degree of biocompatibility and can act as the scaffold for repaired blood vessels or new bone tissues in the healing process of bone damage. ${ }^{2-7}$ However, it lacks the property of osteoinduction, and most HA is not absorbable. DFDBA exhibits quite good osteoinduction characteristics but lacks the scaffolding effect for space maintenance. In addition, DFDBA is made from human cadavers, which limits its source and represents a risk of one in 6 million for infection by human immunodeficiency virus (HIV) and other diseases. ${ }^{13,14}$ Thus, to seek other desirable bone graft materials made from animals or artificial

* To whom correspondence should be addressed. Tel.: +886-22392-2300. Fax: +886-2-2392-2310. E-mail: d49213003@ym.edu.tw.

$\dagger$ National Yang-Ming University.

Taipei Veterans General Hospital.

$\S$ National Taiwan University.

"Industrial Technology Research Institute.

$\perp$ Taipei Medical University. materials that can simultaneously present the characteristics of biocompatibility, osteoconduction, and osteoinduction and are appropriate for larger defects with better safety and lower costs is the primary goal. One of the examples is True Bone Ceramics. ${ }^{15,16}$

In this study, we discuss the use of supercritical fluid (SCF) technology in the fabrication and processing of porcine-derived alloplastic bone graft materials. SCFs exhibit many favorable properties, such as gaslike viscosity, liquidlike density, high diffusion coefficient, and gaslike zero surface tension. ${ }^{17,18} \mathrm{~A}$ very interesting example of the application of SCF technology is that Danish wood industry uses supercritical carbon dioxide $\left(\mathrm{SCCO}_{2}\right)$ to impregnate antiseptic into deep wood to enhance resistance from corrosion, termites, and mosquitos. ${ }^{18}$

Recently, SCF technology has also expanded into the food and drug manufacturing industries. ${ }^{17-19}$ However, the number of studies on the application of SCF technology in graft materials is very limited. Fages et al., a French research team, utilized $\mathrm{SCCO}_{2}$ to remove lipid content of cancelous bone in sheep spine. ${ }^{20}$ Hydrogen peroxide $\left(\mathrm{H}_{2} \mathrm{O}_{2}\right)$, sodium hydroxide $(\mathrm{NaOH})$, sodium dihydrogen phosphate $\left(\mathrm{NaH}_{2} \mathrm{PO}_{4}\right)$, and ethanol have also been utilized for a series of processing to remove partial protein ingredients of sheep bone and decrease the antigenicity. ${ }^{21}$ They confirmed that such ovine-derived materials exhibit good biocompatibility and osteoconduction by a series of in vivo tests. ${ }^{22-24}$ Afterward, they developed a commercial commodity, called OXBONE. ${ }^{23,24}$ However, in their studies, $\mathrm{SCCO}_{2}$ was used only to remove lipid content, whereas other advantages and characteristics of SCF, such as the high penetrability and the high dissolving power, were not exploited. Moreover, the procedures in Fages' studies were quite complicated. Thus, in 
our laboratories, we have utilized $\mathrm{SCCO}_{2}$ with another method in this study to prepare novel alloplastic bone grafts from porcine vertebrae.

The aim of this paper is to report the qualitative evaluation of the biocompatibility of novel porcine-derived alloplastic bone grafts that were prepared by $\mathrm{SCCO}_{2}$ with or without a specific modifier or a specific cross-linker. The modifier is used to modify the properties of the SCF, such as miscibility and polarity, and the cross-linker is used to process the samples in the SCF. For simplicity and qualitative consideration, biocompatibility was evaluated by means of cytotoxicity and cytocompatibility tests. ${ }^{25,26}$ Level I cytotoxicity tests, i.e., MTT assays, were performed using mouse 3T3 fibroblasts and human MG63 osteoblastic-like cells, to assess the influence of the material extracts obtained from culture medium on mitochondria (indirect tests). Cytocompatibility was determined by studying the proliferation of MG63 cells cultured directly onto novel alloplastic bone grafts (direct tests).

\section{Materials and Methods}

2.1. Materials and Sample Preparation. The cancelous portion of porcine vertebrae, cut into fragments of $10 \times 5 \times 5$ mm size, was used as the experimental sample in this study. An SCF extraction system with a 10-mL cartridge body (SFX 210 model, ISCO Inc., Lincoln, NE) was used to process the fragments of porcine vertebrae. The experimental samples were divided into four experimental groups of novel alloplastic bone grafts. The first group (no. 1) was processed statically by $\mathrm{SCCO}_{2}$ at $45{ }^{\circ} \mathrm{C}$ and $40 \mathrm{MPa}$ for $10 \mathrm{~min}$ and then dynamically at a flow rate of $5 \mathrm{~mL} / \mathrm{min}$ for $30 \mathrm{~min}$. The second group (no. 2) was processed statically at $45^{\circ} \mathrm{C}$ and $40 \mathrm{MPa}$ for $10 \mathrm{~min}$ by $\mathrm{SCCO}_{2}$ to which ethanol had been added as a specific modifier at a molar fraction of $10 \%$ and then processed dynamically at a flow rate of $5 \mathrm{~mL} / \mathrm{min}$ for $30 \mathrm{~min}$. The third group (no. 3) was processed statically at $45^{\circ} \mathrm{C}$ and $40 \mathrm{MPa}$ for $10 \mathrm{~min}$ by $\mathrm{SCCO}_{2}$ to which $10 \%$ molar fraction of ethanol and $0.5 \%$ molar fraction of glutaraldehyde (GA) had been added as a specific modifier and a specific cross-linker, respectively, and then processed dynamically at a flow rate of $5 \mathrm{~mL} / \mathrm{min}$ for $30 \mathrm{~min}$. The fourth group (no. 4) was processed statically under subcritical carbon dioxide conditions at $31{ }^{\circ} \mathrm{C}$ and $7 \mathrm{MPa}$ for $10 \mathrm{~min}$ and then dynamically at a flow rate $5 \mathrm{~mL} / \mathrm{min}$ for 30 min. The prepared samples were examined by scanning electron microscopy (SEM).

2.2. Cell Cultures. MG63 osteoblast-like cells [American Type Culture Collection (ATCC), Manassas, VA], originally isolated from a human osteosarcoma, and mouse fibroblast 3T3 cell line (ATCC) were cultured in Dulbecco Modified Eagle's Medium (DMEM, Sigma), containing penicillin/streptomycin $(100 / 100 \mathrm{U})$, amphotericin B $(2.5 \mu \mathrm{g} / \mathrm{mL})$, and gentamycin $(100$ $\mu \mathrm{g} / \mathrm{mL}$ ), supplemented with $10 \%$ fetal calf serum (Biological Industries), and kept at $37{ }^{\circ} \mathrm{C}$ in an atmosphere of $5 \% \mathrm{CO}_{2}$ and $99 \%$ humidity. Media were changed every 3 days.

2.3. Biocompatibility Studies. The toxicity levels deriving from the four groups of novel samples were investigated through indirect tests. In cytotoxicity tests, the viability and metabolic activity of cells previously incubated with material extracts were checked by the 3-(4,5-dimethilthiazol-2-yl)-2,5-diphenyltetrazolium bromide (MTT) assay. MTT is taken up into the mitochondria by viable cells. ${ }^{25,26}$

Extracts for indirect tests were obtained from materials under standardized conditions (ISO 10993-5). The materials were immersed in a complete culture medium for $120 \mathrm{~h}$ at $37{ }^{\circ} \mathrm{C}$ without agitation. The ratio between the sample surface and the volume of the extraction vehicle was $2 \mathrm{~cm}^{2} / \mathrm{mL}$. A negative control was represented by the extraction vehicle with no material, and a positive control by the same medium containing a $0.1 \%$ phenol solution. The pure extracts $(100 \%)$ of four groups of samples were tested with both MG63 cells and 3T3 cells, and the no. 3 samples and their $50 \%, 25 \%$, and $12.5 \%$ dilutions were tested with MG63 cells. The pure extracts and the 50\%, $25 \%$, and $12.5 \%$ dilutions (prepared using the complete culture medium) were added to cells that had been seeded in 96 multiwell plates $24 \mathrm{~h}$ earlier. After 3 days of incubation, the viability and metabolic activity of the cells were tested by MTT assays. $^{25,26}$ The medium was replaced with $50 \mu \mathrm{L} /$ well of MTT solution $(1 \mathrm{mg} / \mathrm{mL}$ in culture medium without phenol red). After $4 \mathrm{~h}$ of incubation at $37^{\circ} \mathrm{C}$, the solution was removed; $100 \mu \mathrm{L} /$ well of DMSO was added; and after 10 min of slow shaking, the absorbance at $540 \mathrm{~nm}$ was measured with a microplate spectrophotometer (SUNRISE, Austria).

For the direct contact method, samples were located in a sixmultiwell plate and then seeded at a cell density of $2.0 \times 10^{4}$ cells $/ \mathrm{cm}^{2}$ in the complete culture medium containing sodium ascorbate $(50 \mu \mathrm{g} / \mathrm{mL})$ and $\beta$-glycerolphosphate $(10 \mathrm{mM})$. The fourth group of samples and the polystyrene standard culture plate (ps) were used as controls. Onto every sample, $200 \mu \mathrm{L}$ of a cell suspension was applied with great care, and the cells were allowed to attach for $2 \mathrm{~h}$ to the underlying substrate, after which $3.5 \mathrm{~mL}$ of culture medium was also added carefully. The amount of cells seeded on the polystyrene control $\left(1.9 \times 10^{5}\right.$ cells $)$ was such as to obtain the same cell density used for the test samples. After 10 days of incubation, cell proliferation was evaluated by MTT assay. Three replicates were performed for each experiment, and the absorbance values of the assay were normalized per unit of area of $1 \mathrm{~cm}^{2}$.

2.4. Statistical Analysis. Results are expressed as mean value \pm standard deviation for each group of samples. After the assessment of significant differences by one-way analysis of variance (ANOVA), differences among groups were assessed with a $t$-test analysis by a two-population comparison. Statistical significance was considered at a probability $p<0.05$.

\section{Result}

In the present work, cytotoxicity tests of novel alloplastic bone graft samples were performed using both human MG63 osteoblastic-like cells and mouse fibroblast 3T3 cell line. SEM images of the four groups of samples reveal a porous structure and no significant differences among the samples. Figure 1 shows the structure of the no. 3 samples obtained by SEM. The results of MTT assays are shown in Figures 2 and 3, and Figure 4 shows the results for pure extracts and different dilutions. In addition, Figure 5 shows the results of cytocompatibility tests by the direct contact method. Statistical analysis revealed that there were significant differences $(p<0.001)$ between the proliferation behaviors of osteoblastic cells on the various materials.

In Figure 2, the absorbance values obtained for material extracts of four groups of novel samples were found to be significantly higher $(p<0.05)$ than that of the positive control (phenol solution). The absorbance values of the no. 3 samples exhibited no significant differences $(p>0.05)$ from those of the negative control (medium without extracts), and both were found to exhibit significantly higher $(p<0.05)$ values than the nos. 1, 2, and 4 samples. The absorbance values of the no. 2 samples also were found to be significantly higher $(p<0.05)$ than those of the no. 4 samples, whereas there were no 


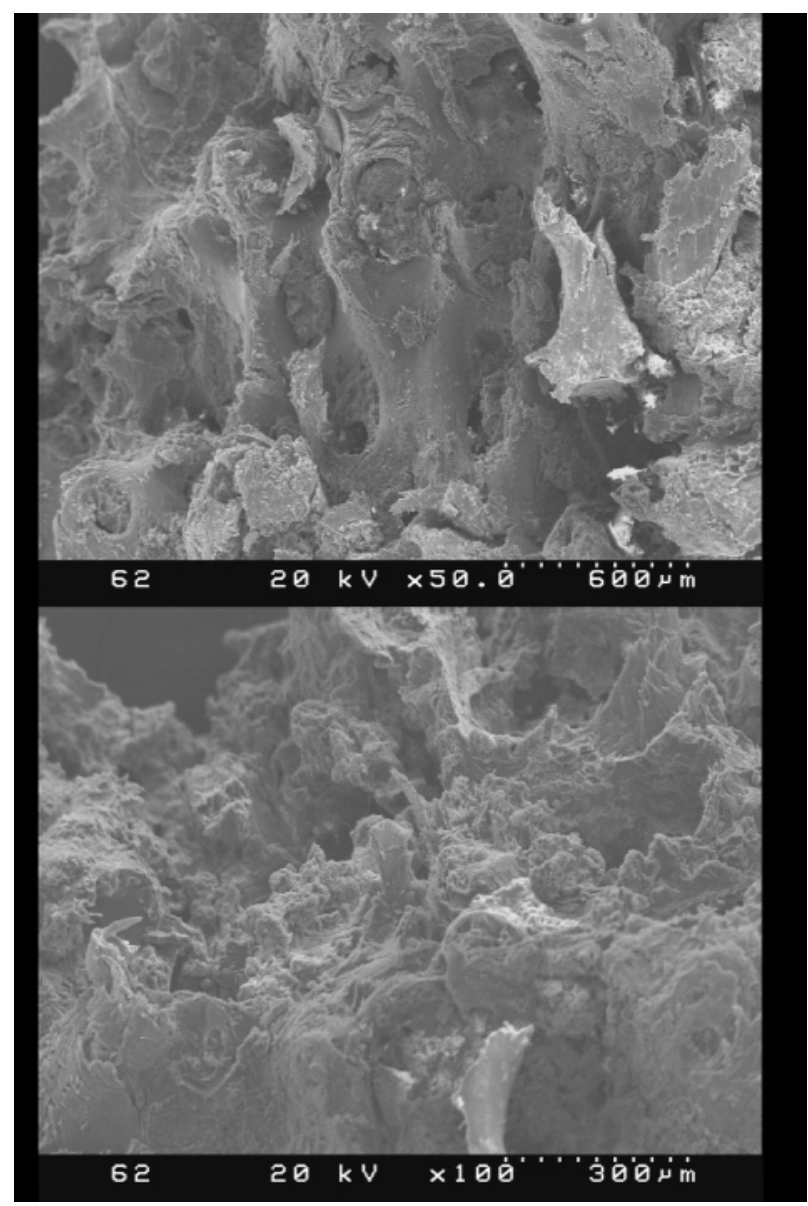

Figure 1. SEM of the no. 3 samples at (top) 50 and (bottom) $100 \times$ magnification. The structure is porous. A porous structure is favored by osteoblasts according to the literature. ${ }^{11}$

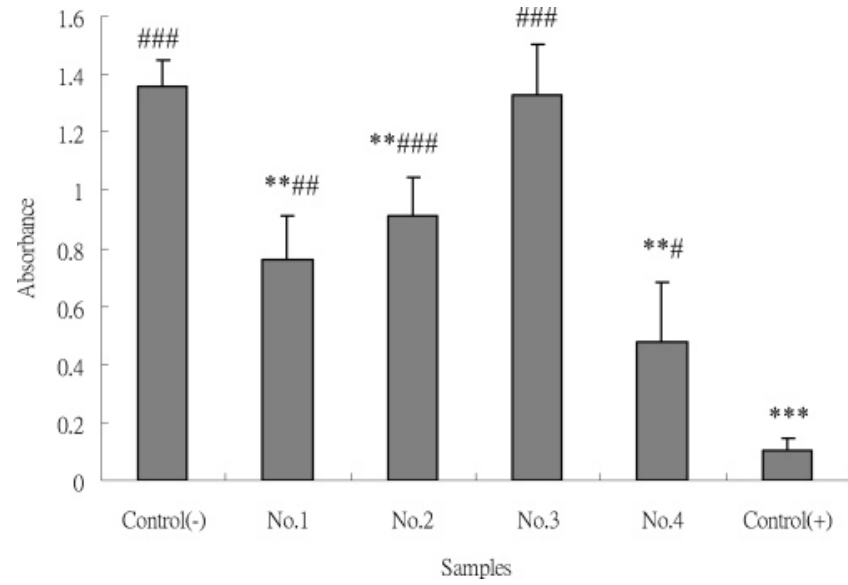

Figure 2. Cytotoxicity evaluation of four groups of novel samples using human MG63 osteoblast-like cells by means of MTT assays. The absorbance values are proportional to the number of viable cells. The negative control $[\operatorname{control}(-)]$ is represented by the extraction vehicle with no material, and the positive control [control $(+)]$ is represented by the same medium containing a $0.1 \%$ phenol solution. Difference from control(-): *, $p<$ $0.05 ; * *, p<0.01 ; * * *, p<0.001$. Difference from control(+): \#, $p<$ $0.05 ; \# \#, p<0.01 ; \# \#, p<0.001$.

significant differences $(p>0.05)$ between the values for the nos. 1 and 2 samples and between those for the nos. 1 and 4 samples.

In Figure 3, the absorbance values obtained for material extracts of four groups of test samples were found to be significantly higher $(p<0.05)$ than that of the positive control. The absorbance values of the no. 3 samples exhibited no

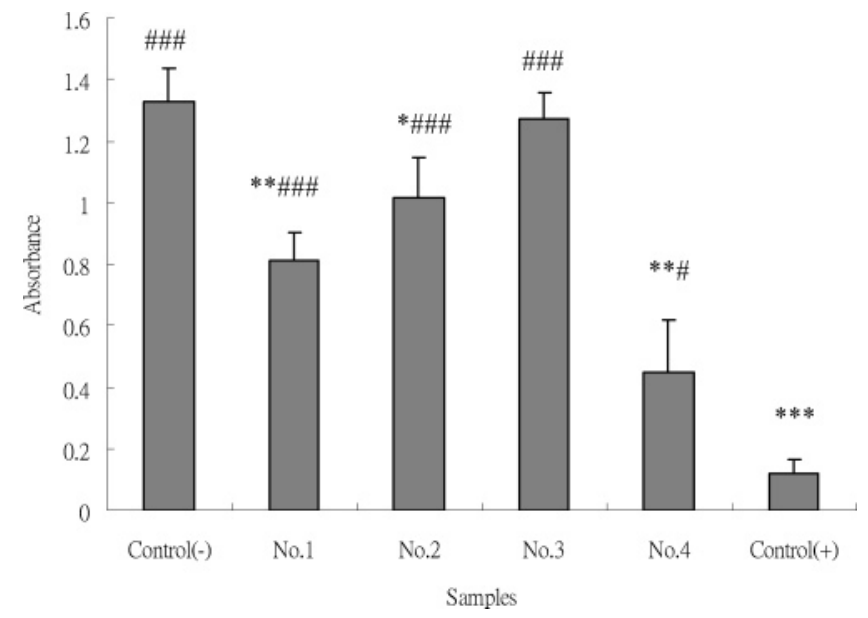

Figure 3. Cytotoxicity evaluation of four groups of novel samples using mouse fibroblast 3T3 cell line by means of MTT assays. The absorbance values are proportional to the number of viable cells. The negative control $[\operatorname{control}(-)]$ is represented by the extraction vehicle with no material, and the positive control $[\operatorname{control}(+)]$ is represented by the same medium containing a $0.1 \%$ phenol solution. Difference from control $(-)$ : *, $p<$ $0.05 ; * *, p<0.01 ; * * *, p<0.001$. Difference from control $(+)$ : $\#, p<$ $0.05 ; \# \#, p<0.01 ; \# \# \#, p<0.001$.

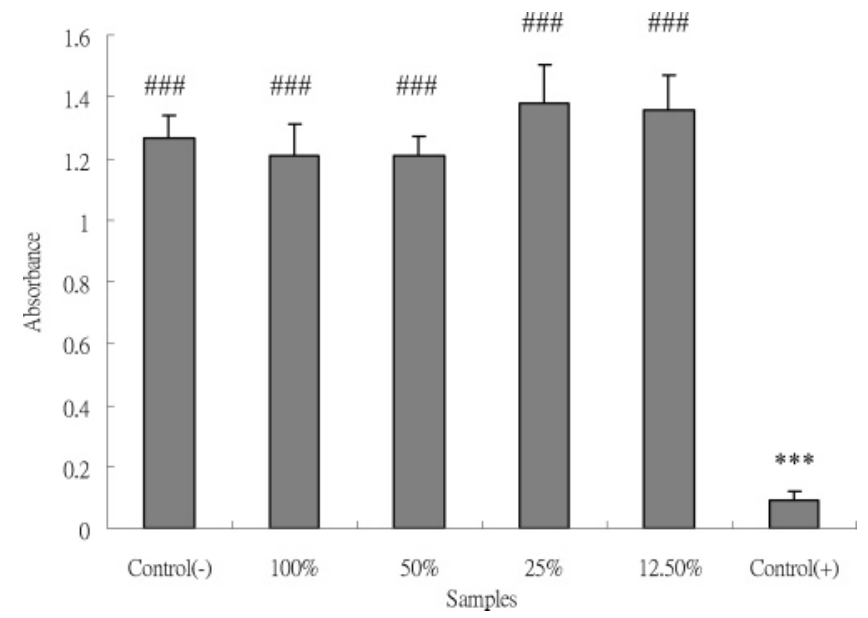

Figure 4. Cytotoxicity evaluation of the no. 3 samples using human MG63 osteoblast-like cells at different extract dilutions by means of MTT assays. The absorbance values are proportional to the number of viable cells. The negative control $[\operatorname{control}(-)]$ is represented by the extraction vehicle with no material, and the positive control $[\operatorname{control}(+)]$ is represented by the same medium containing a $0.1 \%$ phenol solution. Difference from control $(-)$ : $*, p<0.05 ; * *, p<0.01$; ***, $p<0.001$. Difference from control(+): \#, $p<0.05$; \#\#, $p<0.01$;\#\#, $p<0.001$.

significant differences $(p>0.05)$ from those of the negative control, and the values of both were significantly higher $(p<$ 0.05 ) than those of the nos. 1, 2, and 4 samples. The absorbance values of the no. 1 samples showed no significant differences $(p>0.05)$ from those of the no. 2 samples, and the values of both were significantly higher $(p<0.05)$ than those of the no. 4 samples.

In Figure 4, the absorbance values obtained for pure material extracts and different dilutions of the no. 3 samples are shown to be significantly higher $(p<0.05)$ than that of the positive control and comparable to that of the negative control. No significant differences were observed among the dilutions of extracts and the high-concentration extract solution (100\%). The extracts did not induce a cell viability reduction or an inhibition of cell growth, indicating that they have no toxic effects.

In the direct contact method, MG63 cells were seeded directly onto the test materials, and their viability and proliferation were assessed after 10 days of incubation by the MTT test (Figure 


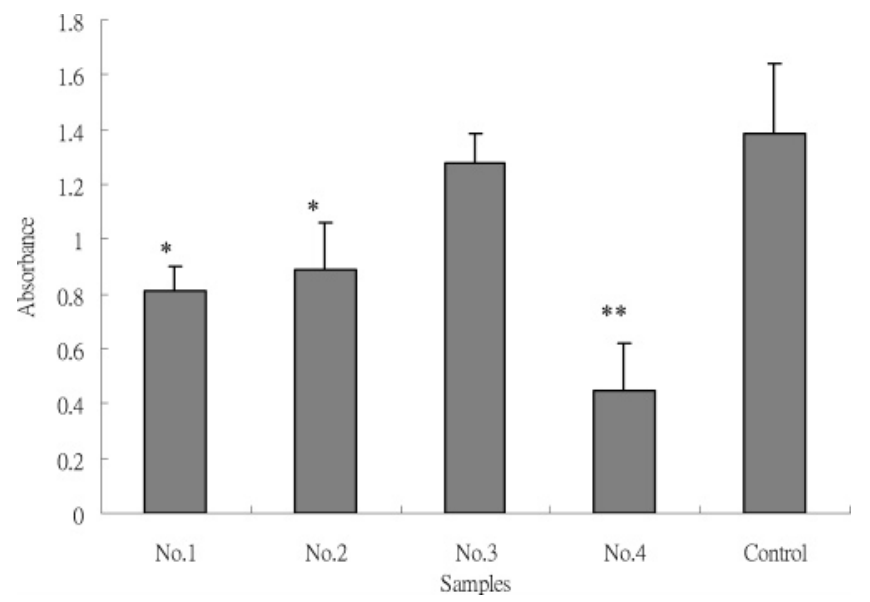

Figure 5. Evaluation of cell viability estimated by the MTT assay of MG63 osteoblast-like cells cultured on four groups of samples after 10 days of incubation, compared with a control represented by polystyrene. The absorbance values, normalized per unit of area of $1 \mathrm{~cm}^{2}$ and reported as the mean \pm standard deviation, are proportional to the number of viable cells. The means of the no. 3 samples and the control are not significantly different. Difference from control: $*, p<0.05$; **, $p<0.01$.

5). A polystyrene standard culture disk was used as a control for comparison. Statistical analysis revealed that there were no significant differences between the proliferation of osteoblastic cells on the no. 3 samples and the control, whereas the values of both were significantly higher $(p<0.05)$ than those of the nos. 1, 2, and 4 samples. The values of the no. 2 samples exhibited no significant differences $(p>0.05)$ from those of the no. 1 samples, whereas the values of both were significantly higher $(p<0.05)$ than those of the no. 4 samples.

To summarize, the results obtained from the cytotoxicity tests of the no. 3 samples performed on both cell types and the cytocompatibility test were in good agreement, whereas the results for the other samples were not.

\section{Discussion}

The results of the cytotoxicity tests suggest that extracts obtained from the no. 1 samples might affect cell viability and proliferation, indicating that porcine-derived bone grafts processed by only $\mathrm{SCCO}_{2}$ are not sufficiently biocompatible. $\mathrm{SCCO}_{2}$ can penetrate into the deep content of the samples and remove the soluble substances, i.e., lipid and water. The great drying effect can affect the activity of proteins through removal of the bound water in the protein structure. ${ }^{27,28}$ Thus, the cytotoxicity of $\mathrm{SCCO}_{2}$-treated samples was lower than that of subcritically prepared no. 4 samples.

The results for the no. 2 samples showed better mean values than the no. 1 samples, but the standard deviation values revealed no significant difference, indicating that the no. 2 samples might also affect cell viability and proliferation and might not be sufficiently biocompatible with the tested cells. This suggests that the porcine-derived bone grafts processed by $\mathrm{SCCO}_{2}$ and $10 \%$ ethanol might not effectively reduce the cytotoxicity of the bone graft. Ethanol is commonly used as a disinfectant and antiseptic agent and might denature proteins at high concentration. Lipids, esters, alcohols, aldehydes, and ketones are quite soluble in $\mathrm{SCCO}_{2}$, whereas proteins, glycosides, sugars, fruit acids, starch, and mineral salts are rarely soluble because of their polarity. ${ }^{17,18}$ However, adding modifiers into $\mathrm{SCCO}_{2}$ might change its miscibility. Ethanol is one of the most commonly used modifiers. Thus, adding ethanol into $\mathrm{SCCO}_{2}$ was expected to increase the miscibility of lipids and residual organic chemicals to reduce the cytotoxicity. The results of indirect tests reveal that the processed novel bone grafts are not yet sufficiently biocompatible with the tested cells. Therefore, an additional modification is needed.

When both types of cells were seeded directly onto the no. 3 samples, cell proliferation occurred equally well as it did with the negative control, revealing no significant differences. These results indicate that the extracts obtained with the no. 3 samples do not affect cell viability and proliferation, indicating that the no. 3 samples have quite good affinity and biocompatibility with both the MG63 osteoblastic-like cells and the mouse fibroblast $3 \mathrm{~T} 3$ cells. The results for the pure extracts and the dilutions of the no. 3 samples in indirect cytotoxicity tests also confirmed their good biocompatibility. These results indicate that porcinederived bone grafts processed by $\mathrm{SCCO}_{2}$ and $10 \%$ ethanol and $0.5 \%$ GA have quite good biocompatibility with the tested cells. This improvement might be due to the addition of GA. GA is commonly and clinically used as a disinfectant and antiseptic agent at under $2 \%$ concentrations for at least $10 \mathrm{~min}$ for viruses. ${ }^{29}$ In addition, GA is commonly used as a cross-linking agent for proteins, especially collagens. ${ }^{25,30,31}$ Thus, the addition of GA into $\mathrm{SCCO}_{2}$ was expected to cross-link partial proteins of the porcine-derived bone graft to reduce its antigencity and increase its biocompatibility. The static $\mathrm{SCCO}_{2}$ processing procedure was expected to help carry GA into the deep content and then cross-link proteins, whereas the dynamic procedure was expected to remove all of the undesired residual GA and chemical agents. Hence, the cytotoxicity of GA was not found in the tests, and the results revealed good biocompatibility. The better performances of these GA-treated grafts with respect to those of the untreated grafts can be explained by the characteristics of the chemical composition differences that affect biological properties. Previous work in our laboratory revealed that the composition of a novel bone graft prepared under the same conditions as the no. 3 samples was $64.5 \pm 0.5 \%$ mineral phase, $28.5 \pm 0.5 \%$ proteins, and $0.4 \pm 0.1 \%$ lipids, ${ }^{32}$ in which the constituent ratio of minerals and proteins is similar to that of the original bone. ${ }^{11}$ SEM images of treated samples revealed that the structure is porous. A porous structure is favored by osteoblasts. ${ }^{11}$ Hence, an additional examination of the exact porosity and composition of the prepared samples is needed in the future. In addition, the exact differences in the physical and chemical properties of the prepared samples have to be evaluated.

The no. 4 samples were processed under subcritical conditions statically for $10 \mathrm{~min}$ and then dynamically for $30 \mathrm{~min}$. This group is also a control group with respect to the other three groups of supercritically treated samples. The results revealed a quite undesirably high cytotoxicity to the tested cells. This outcome is thought to be due to the poor miscibility of the subcritical $\mathrm{CO}_{2} \cdot{ }^{17,18}$

In the Fages et al. study, cancelous bone of vertebrae from a single sheep was treated with $\mathrm{SCCO}_{2}$ for the removal of the lipid content. The operating conditions were as follows: $\mathrm{CO}_{2}$ flow rate, $2 \mathrm{~kg} / \mathrm{h}$; pressure, $25 \mathrm{MPa}$; extraction temperature, 50 ${ }^{\circ} \mathrm{C}$; time, $10 \mathrm{~min} / \mathrm{g}$ of bone. After being unloaded from the extraction vessel, samples were immersed in a $35 \% \mathrm{H}_{2} \mathrm{O}_{2}$ solution at $40{ }^{\circ} \mathrm{C}$ for $24 \mathrm{~h}$ and then treated in $\mathrm{NaOH}$ solution $(1$ M) for $1 \mathrm{~h}$ at room temperature. They were buffered for 15 min in $\mathrm{NaH}_{2} \mathrm{PO}_{4}(12 \mathrm{~g} / \mathrm{L})$, before being rinsed for $3 \mathrm{~h}$ in an ethanol solution $(95 \%)$ and then in absolute ethanol for $2 \mathrm{~h}$. The composition of the processed bone was $63.5 \pm 0.5 \%$ mineral phase, $24.43 \pm 0.5 \%$ proteins, and $0.5 \pm 0.01 \%$ lipids. ${ }^{20-24}$ This novel bone graft revealed good compatibility. ${ }^{20-24}$ 
However, SCF was utilized only to remove the lipid content of the sheep bone in the Fages et al. study, and the other characteristics and advantages of SCF were not be completely utilized, for example, the high permeability and the material processing capability. ${ }^{17,18}$

Ethanol is generally added to $\mathrm{SCCO}_{2}$ as a specific modifier. It can change the solubility of polar and nonpolar substance in $\mathrm{SCCO}_{2}$. For example, the solubility of polar substance will increase in the presence of modifier. ${ }^{18} \mathrm{GA}$ is added to $\mathrm{SCCO}_{2}$ as a specific cross-linker. It can cross-link protein content and decrease antigencity and is widely used in the cross-linking of collagens and proteins. ${ }^{25,30,31}$ In addition, GA and ethanol are generally and clinically used for the sterilization and disinfection of instruments. Thus, GA was chosen as the cross-linking agent in this study. Although GA remaining in the collagen material might induce mild cytotoxicity, ${ }^{30,31}$ in the present study, the $\mathrm{SCCO}_{2}$ was able to remove the entire remaining GA in the novel bone grafts by the dynamic process because of the high solubility of $\mathrm{GA}$ in $\mathrm{SCCO}_{2}{ }^{17,18}$

Traditionally, viral inactivation of alloplastic and allogenic bone grafts is generally achieved by $\beta$ or $\gamma$ irradiation, ethylene oxide, or autoclaving. None of these methods have proven to be completely satisfactory, either because of the resistance of some viruses to physicochemical processing or the loss of inductive or mechanical properties of the graft material. ${ }^{33-36}$ In the Fages et al. study, the sterilization and disinfection effect of $\mathrm{SCCO}_{2}$ and the other procedures were evaluated. The results showed that $\mathrm{SCCO}_{2}$ contributed to a 4.02-6.58 logarithmic unit reduction of the viruses, a 4.21-6.6 logarithmic unit reduction of ethanol, and a total reduction of 14.22-24.48 logarithmic units. ${ }^{22}$ In recent years, $\mathrm{SCCO}_{2}$ has been shown to be capable of sterilizing and disinfecting materials and instruments. ${ }^{18,19,37-40}$ One study investigated the inactivation of coronavirus and Japanese encephalitis virus (JEV) with $\mathrm{SCCO}_{2}$ as the disinfection medium, and nearly $100 \%$ of the coronavirus and JEV were killed at $\mathrm{SCCO}_{2}$ conditions of $40{ }^{\circ} \mathrm{C}$ and $16 \mathrm{MPa}$ for $1 \mathrm{~h} .{ }^{38} \mathrm{In}$ addition, some studies have determined that, under adequate conditions, $\mathrm{SCCO}_{2}$ is valid, effective, and efficient for the inactivation of staphylococcus aureus, ${ }^{39}$ the sterilization of bacterial spores, ${ }^{40}$ and the inactivation of viruses. ${ }^{19}$

To summarize, in the present study, $\mathrm{SCCO}_{2}$ combined with a specific modifier, i.e., ethanol, and a specific cross-linker, i.e., GA, was used to prepare novel porcine-derived alloplastic bone grafts that exhibited good biocompatibility with both human MG63 osteoblastic-like cells and mouse fibroblast 3T3 cells. Further investigations are in progress to test these results and to provide a complete interpretation of these events. The efficiency of this process for sterilization and disinfection also must be verified.

\section{Conclusion}

This study of the biocompatibility of novel porcine-derived alloplastic bone grafts, prepared by processing in $\mathrm{SCCO}_{2}$ combined with ethanol and GA, suggests that these porcinederived materials are not cytotoxic and allow the differentiation and proliferation of cells. The good performances of these novel bone grafts can be explained by the characteristics of SCF. Additional advanced in vitro and in vivo studies must still be performed to optimize the process. Therefore, the application of SCF in biomaterials is very promising, and a better understanding of cell-biomaterial interactions and mechanisms can help in the development of more effective alloplastic bone grafts and contribute to bone regeneration and tissue engineering.

\section{Literature Cited}

(1) Younger, E. M.; Chapman, M. W. Morbidity at bone graft donor sites. J. Orthop. Trauma 1989, 3 (3), 192-195.

(2) Salyer, K. E.; Hall, C. D. Porous hydroxyapatite as an onlay bonegraft substitute for maxillofacial surgery. Plastic Reconstructive Surg. 1989, 84 (2), 236-244.

(3) Lin, T. C.; Su, C. Y.; Chang, C. S.; Stereomorphologic observation of bone tissue response to hydroxyapatite using SEM with the EDTA-KOH method. J. Biomed. Mater. Res. 1997, 36, 91-97.

(4) Shu, F. Y., W. Y. J., Studies of the Bone Grafting Materials Based on Collagen Microspheres Containing Hydroxyapatite. Ph.D. Thesis, Institute of Biomedical Engineering, National Yang-Ming University, Taiwan, 1999.

(5) Chang, C. S.; Kao, S. Y. Biomechanical and histological studies of particulate hydroxylapatite implanted in femur bone defects of adult dogs. Int. J. Oral Maxillofac. Surg. 2000, 29, 54-61.

(6) Kao, S. Y.; Iou, G. T.; Lo, W. L.; Chang, Y. L.; Su, C. Y.; Chang, C. S. An overview of retrospective and prospective researches on ceramic bone substitue hydroxylapatite-HA. Chin. Dent. J. 2001, 20, 227-242.

(7) Heise, U.; Osborn, J. F.; Duwe, F. Hydroxyapatite ceramic as a bone substitute. Int. Orthop. 1990, 14 (3), 329-338.

(8) Cheng, C. M.; Chang, C. S.; Su, C. Y. A Steremorphological Study of Bone Regeneration Following Hydroxyapatite or DFDBA Implantation Using with EDTA-KOH Method. M.S. Thesis, Institute of Dental Science, National Yang-Ming University, Taiwan, 1997.

(9) Nyman, S. Bone regeneration using the principle of guided tissue regeneration. J. Periodont. 1991, 18 (6), 494-498.

(10) Li, S. T. Biomaterials (collagen). In The Biomedical Engineering Handbook; Bronzino, J. D., Ed.; CRC Press: Boca Raton, FL, 1995.

(11) Iou, G. T.; Chang, C. S.; Su, C. Y. A Comparative Study on Microcirculatory Changes and Bone Formation of Porous HA-Implanted Cavities or Porous HA/DFDBA-Implanted Cavities by SEM. M.S. Thesis, Institute of Clinical Dental Science, School of Dentistry, National YangMing University, Taiwan, 2002.

(12) Hedner, E.; Linde, A. Efficacy of bone morphogenetic protein (BMP) with osteopromotive membranes - an experimental study in rat mandibular defects. Eur. J. Oral Sci. 1995, 103 (4), 236-241.

(13) Buck, B. E.; Malinin, T. I.; Brown, M. D. Bone transplantation and human immunodeficiency virus. An estimate of risk of acquired immunodeficiency syndrome (AIDS). Clin. Orthop. Relat. Res. 1989, 240, 129-136.

(14) Roder, V. V.; Muller, H. HIV infection in human bone. J. Bone Joint Surg. 1992, 748, 179-80.

(15) Diau, M. H.; Roan, R. T. True Bone Ceramic Bone Grafts. M.S Thesis, Institute of Dental Science, Kaohsiung Medical College, Taiwan, 1998.

(16) Shen, L. C.; Roan, R. T. Critical Sized Defect Repaired with True Bone Ceramic. M.S. Thesis, Institute of Dental Science, Kaohsuing Medical University, Taiwan, 2003.

(17) Tan, S. C. Applications of Supercritical Fluid. Sci. Dev. (NSC) 2002 , $359,12-17$.

(18) 2003 International Conference and Exhibition on the Supercritical Fluid Technology, Taiwan, Oct 2003.

(19) Perrut, M. Applications of Supercritical Fluids in Health Sciences. Presented at the International Symposium on Supercritical Fluids 2005, 2005; Paper 461.

(20) Fages, J.; Marty, A.; Delga, C.; Condoret, J. S.; Combes, D.; Frayssinet, $\mathrm{P}$. Use of supercritical $\mathrm{CO}_{2}$ for bone delipidation. Biomaterials 1994, 15, 650-656.

(21) Frayssinet, P.; Fages, J.; Asimus, E.; Autefage, A. Histological evaluation of xenogeneic bone treated by supercritical $\mathrm{CO}_{2}$ implanted into sheep. J. Mater. Sci. Mater. Med. 1995, 6, 473-478.

(22) Fages, J.; Frayssinet, P. Viral inactivation of bone allografts using supercritical fluid extraction. Presented at the Fifth World Biomaterials Congress, Toronto, Canada, May 29-Jun 2, 1996.

(23) Fages, J., et al. Bone allografts and supercritical processing: effects on osteointegration and vira safety. J. Supercrit. Fluids 1998, 13, 351356.

(24) Frayssinet, P., et al. Histological integration of allogeneic cancelous bone tissue treated by supercritical $\mathrm{CO}_{2}$ implanted in sheep bones. Biomaterials 1998, 19, 2247-2253.

(25) Ramires, P. A.; Milella, E. Biocompatibility of poly(vinyl alcohol)hyaluronic acid and poly(vinyl alcohol)-gellan membranes cross-linked by glutaraldehyde vapours. J. Mater. Sci. Mater. Med. 2002, 13 (1), 119123.

(26) Sbarbati Del Guerra R, Lelli, L.; Tonelli, C.; Trombetta, T. Cascone, M. G.; Taveri, M.; Narducci, P.; Giusti, P. In vitro biocompatibility of fluorinated polyurethanes. J. Mater. Sci. Mater. Med. 1994, 5, 452-456. 
(27) Westhof, E. Water and Biological Macromolecules; CRC Press: Boca Raton, FL, 1996.

(28) Ilias Michalarias, Xiuli Gao, Robert C. Ford, Jichen Li. Recent progress on our understanding of water around biomolecules. J. Mol. Liq. 2005, 117, 107-116.

(29) EC regulatory document. Validation of virus removal and inactivation procedures. Note for guidance, III/8115/89-EN. Biologicals 1992, 20 159.

(30) Ma, T. Y. Studies of the structure and property of genipin- or carbodiimide-cross-linked biological materials. M.S. Thesis, Institute, Chemical Engineering, National Central University, Taiwan, 2000.

(31) Chou, C. C. Studies of the Collagen-Based Wound Dressing. M.S. Thesis, Institute of Biomedical Engineering, National Yang-Ming University, Taiwan, 2001

(32) Ching-Feng Chen, Che-Shoa Chang. Applications of Supercritical Fluid in Bone Graft. In The 3rd International Conference on the Supercritical Fluid Technology; 2004; pp 202-209.

(33) Doherty, M. J.; Mollan, R. A. B.; Wilson, D. J. Effect of ethylene oxide sterilisation on human demineralized bone. Biomaterials 1993, 14, 994.

(34) Campbell, D. G.; Li, P.; Stephenson, A. J. Oakeshott RD. Sterilisation of HIV by gamma irradiation: a bone allografe model. Int Orthop. 1994, 18, 172.

(35) Sugiura, H.; Sato, K.; Rong, Y.; Yamamura, S.; Nakanishi, K.; Ito, Y. A mechanical and biological study of surface demineralized and heattreated cortical bone. Eur. J. Exp. Musculoskel. Res. 1994, 3, 157.
(36) Hallfeldt, K. J.; Stutzle, H.; Puhlmann, M.; Kessler, S.; Schweiberer, L. Sterilisation of partially demineralized bone matrix: The effects of different sterilisation techniques on osteogenic properties. J. Surg. Res. 1995, 59,614 .

(37) 2004 International Conference and Exhibition on the Supercritical Fluid Technology, Taiwan, Nov 2004.

(38) Chen, Y.-T.; Chiu, Y.-H.; Chen, C.-M.; Chang, M.-Y. Inactivation of Coronavirus and JEV with Supercritical Carbon Dioxide. In The $3 \mathrm{rd}$ International Conference on the Supercritical Fluid Technology; 2004; pp 197-201.

(39) Van Ginneken, L.; Van Roy, S.; Willems, L.; Kathy Elst, B.; De Cleen Lodewijckx, M. Inactivation of Staphylococcus aureus in Liquid Whole Egg Product by Means of High-Pressure Carbon Dioxide. Presented at the International Symposium on Supercritical Fluids 2005, 2005; Paper 395.

(40) Zhang, J.; Gleason, C.; Dalal, N.; Burrows, S.; Matthews, M.; Fox, A.; Fox, K.; Price, R. L.; Davis, J.; Hemmer, J.; LaBerge, M.; Drews, M.; Mechanisms of Supercritical Carbon Dioxide Sterilization of Bacterial Spores. Presented at the International Symposium on Supercritical Fluids 2005, 2005; Paper 241

Received for review June 15, 2005 Revised manuscript received November 9, 2005 Accepted November 11, 2005

IE0507169 